\title{
Development and Application of a Farmland Test Data Processing System Designed for Wireless Sensor Network Applications
}

\author{
Jinqiu Zou ${ }^{1,2}$, Qingbo Zhou ${ }^{1,2}$, Peng Yang ${ }^{1,2}$, and Wenbin $\mathrm{Wu}^{1,2, *}$ \\ ${ }^{1}$ Key Laboratory of Resources Remote Sensing and Digital Agriculture, \\ Beijing 100081, China \\ ${ }^{2}$ Institute of agricultural resources and regional planning, \\ Chinese Academy of Agricultural Sciences, Beijing 100081, China \\ \{zoujq, zhouqb, yangp, wwb\} @mail. caas. net.cn
}

\begin{abstract}
Data collected by measuring farmland environmental indicators are vital sources of agricultural information. Wireless sensor networks (WSNs) have been employed to acquire stable and real time farmland environment data. WSNs, recognized as one of the latest development trends, recently attracted widespread attention and application due to their relative economy, stability, and sophistication. In this study, based upon a large amount of WSNs-obtained data, Microsoft Visual Studio 2005 and ESRI ArcGIS Engine 9.3, amongst others, were utilized to develop a farmland test data processing system, resolve information storage and utilization problems and conduct system applications. The data processing software consisted of four modules: data receiving conversion, database maintenance management, data browsing analysis and the generation and application of data spatialization outputs. Specifically, the data receiving conversion module was mainly responsible for converting raw data acquired by WSNs into standard database outputs, including the automatic reception of measured value, error correction and alerting the observer to abnormal data. The Database maintenance management module's primary functions were the generation and maintenance of metadata generated from stored data, authentic data enquiry, display and analysis. The generation and application of data spatialization products module was principally for the spatial expansion application of measured data, including space-time interpolation and conversion. The system underwent pilot scale testing and improvement at the same time as undertaking the processing of real data collected by WSNs deployed throughout the Hebi test zone. The observed results revealed that the system was able to complete real-time conversion and management of field measured data. In addition, it has several advantages, such as, excellent stability, perfect functionality and a convenient human - machine interface.
\end{abstract}

Keywords: Wireless sensor networks (WSNs), Measured value, Monitoring.

Corresponding author.

D. Li and Y. Chen (Eds.): CCTA 2011, Part I, IFIP AICT 368, pp. 169-179, 2012.

(C) IFIP International Federation for Information Processing 2012 


\section{Introduction}

Crop farmland ecological environment indicators mainly consist of soil temperature and humidity, air temperature and humidity, photosynthetically active radiation and leaf area index, all of which are indispensable data when assessing an area's suitability for agriculture[1]. In addition, these parameters are strongly linked to crop growth, which is closely correlated with final crop yield and agricultural production efficiency. How best to acquire accurate farmland environmental and soil data and map any space-time dynamic changes that may occur has caught the attention of agriculture departments and scientists both at home and abroad. Some studies looking at information acquisition tools and precision evaluation have been undertaken[2]. Farmland information acquisition tools in particular have undergone constant innovation and change, evolving from artificial spot observations towards assistant observation by remote sensing technology or short-distance wireless collection based upon a PDA and the mobile phone. To date, this tool has developed into a field information collection system integrating accurate positioning and farmland WSNs incorporating the analysis and visualization of field data[3].

New generation WSNs[4] are an integrated technology using computer science techniques, MEMS sensing techniques, network communication techniques and embedded systems. They represent the main trends in the post-PC era for information science technology and also exist at the leading edge of current information science technology. WSNs serve as an important technology that automatically records a range of different data from the physical world. WSNs have become one of the most appealing and vibrant research fields and are regarded as a great opportunity for information technology and application due to their low deployment and maintenance costs, convenient upgrades, and greatly improved mobility that requires no cable connections[5-8]. By installing humidity sensors, temperature sensors, photosensors etc, beneath the observational zone, various farmland data can be directly acquired or inverted and then the data can be automatically transmitted to a laboratory that can process and analyze the observational data automatically collected by the WSNs. This effectively overcomes the drawbacks in traditional farmland environmental monitoring and investigation, and satisfies the application information requirements of modern agriculture[9-11].

WSNs technology has made substantial progress and it now plays a vital role in farmland information data gathering. Present research is mainly inclined to a few front end studies, such as, farmland information collection and information transmission. However, little emphasis has been placed upon back end studies including processing and analysis of the data collected. This study is designed to explore possible application methods for the storage, enquiry, browsing and analysis of measured data based upon the WSN platform. Microsoft Visual Studio 2005 and ESRI ArcGIS Engine 9.3 were utilized to develop a farmland observation data system, integrate the data flow process and the data analysis module to ensure effective application of any data collected. Data obtained by the WSNs was sourced from the sensing network deployed at Hebi city in Henan province which covers a $150 \mathrm{~km}^{2}$ area of the Institute of Natural Resources and Regional Planning, Chinese 
Academy of Agricultural Sciences. Thirty-five nodes were placed and a frame of data was collected every $10 \mathrm{~min}$ which was then transmitted back to the laboratory via GPRS.

\section{Structure and Function Module of the Processing System}

This system aimed to resolve the problems involved in storage and application of WSNs acquired data. To achieve this goal, information processing technologies need to be analyzed to resolve the problems that are associated with, for example: WSNsbased data transmission, current background organization and data application modes. The system must be established under the principles of openness, high-efficiency, and safety[12-13].The system structure is shown in Fig.1.

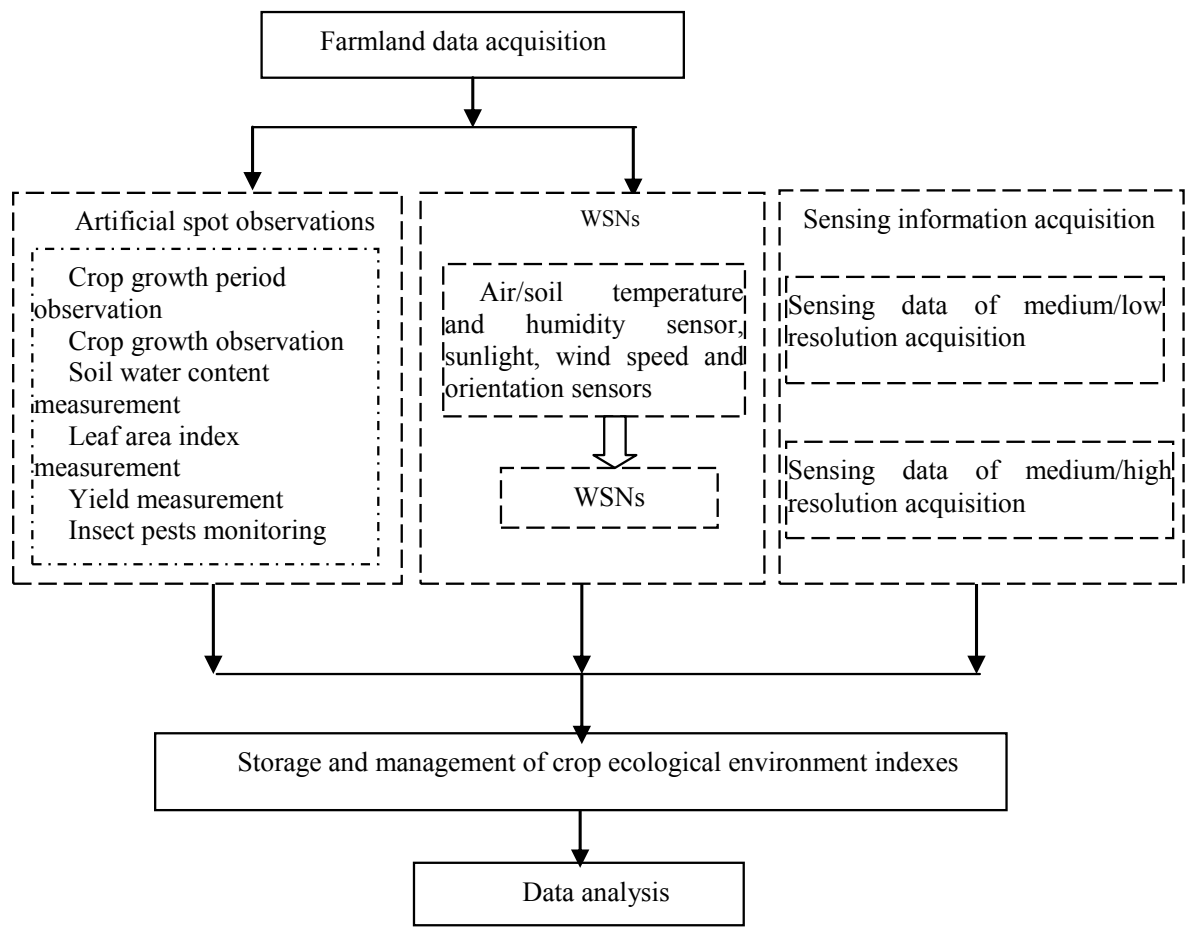

Fig. 1. System structure module scheme

Through requirement analysis and data flow abstraction, the system was divided into 4 modules: data receiving conversion, database maintenance management, data browsing analysis and generation and application of data spatialization outputs. Each module was connected via data utilization flow and each served a specific function forming an integrated data processing system which transformed raw data into various outputs. The system function module scheme is shown below in Fig.2: 


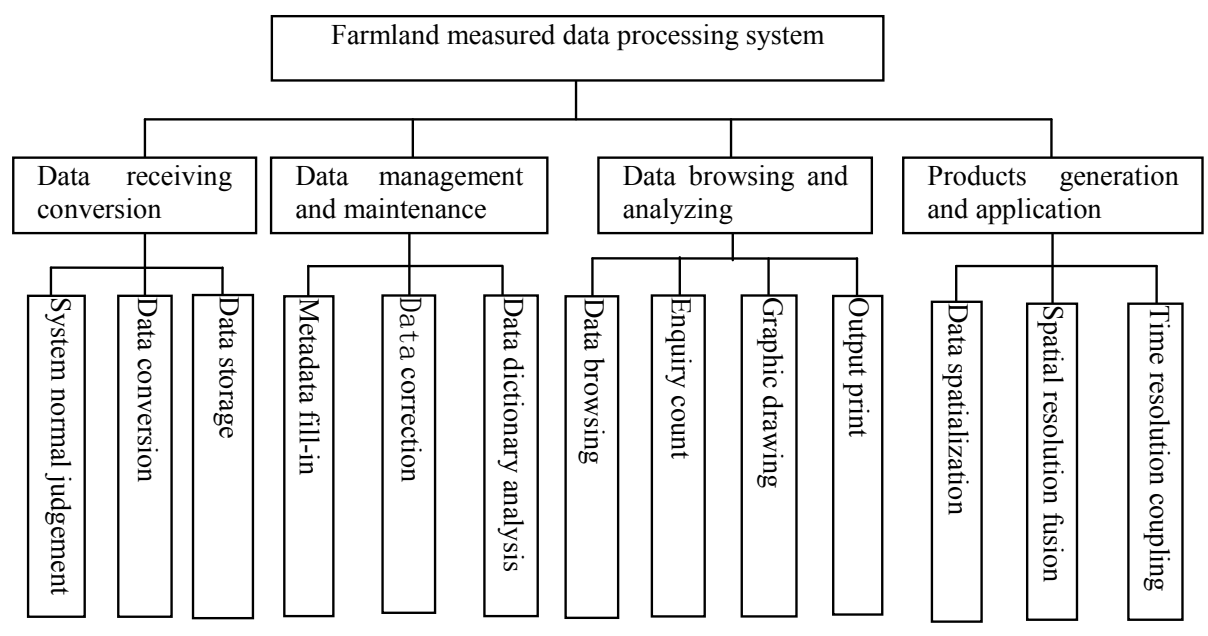

Fig. 2. System function module scheme

\subsection{Data Receiving Conversion Module}

This module received the WSNs data, and served as the data entry portal for the whole system. The main functions included: automatically converting WSN-obtained binary file data into tables in a standard database, embracing automatic operational control of the storage module, acquisition synchronization of the WSNs and the automatic capture and highlighting of abnormal conditions. The WSNs performed long-term operations with no interruption and thereafter the module exhibited stable operation. Considering the flaws existing in machine servers and the network environment, unexpected abnormal errors may arise, such as server breakdown and the system halting and the computer restarting. These results suggest that this module

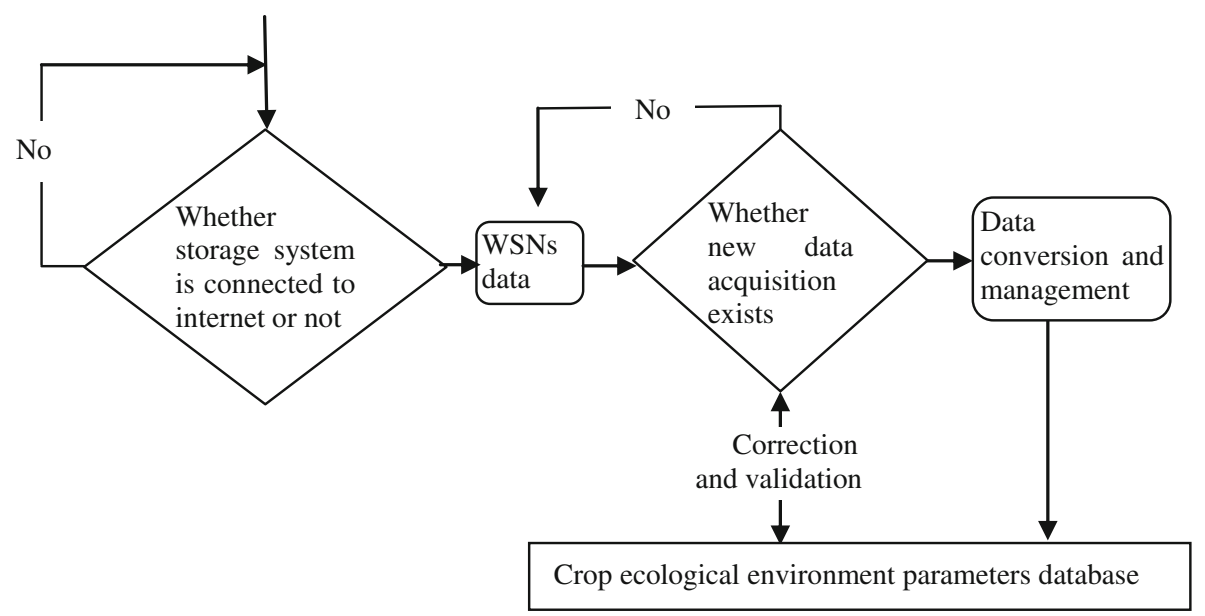

Fig. 3. Data reception conversion module scheme 
should be able to automatically detect these abnormal conditions and ensure a synchronized link between the local system and WSNs by identifying the underlying cause, auto operation, and network registration. In addition, data remains and losses may be caused by these abnormal conditions. Therefore, this module should evaluate data consistency to avert repeated data storage and data loss. The function module is illustrated as follows Fig.3 .

\subsection{Functionality of the Database Management and Maintenance Module}

This module was mainly designed to record and generate metadata for each indicator measured to complete the fusion between this data set and other data sets including addition and registration of time-space related information. Furthermore, this module was also responsible for revising abnormal values to guarantee that all the observational data stored in the database were consistent with conventional thresholds. When undertaking the database design, metadata technology and a doublebackup mechanism were taken into account in order to define an open, standard and integrated series of metadata information for data sets. Metadata was produced while performing data storage and provided a basis for subsequent data enquiry and analysis. Double backups were carried out in the database, one backup served for storing data directly originating from the data receiving conversion module and the second backup was set for qualified standard data after undergoing correction processing.

\subsection{Functionality of the Data Browsing and Analysis Module}

This module performed enquiry, browsing, statistical counts and graphics drawing to stored collected data including some fundamental functions such as, storage, enquiry, printing, data analysis and statistics. It also embraced multiple statistical schemes according to time, node, and location. Statistical methods included maximum, minimum and mean values together with frequency figures. Graphics drawing included various diagrams according to time, node, and index type, etc and drawing methods included line graphs, bar graphs, and pie charts.

\subsection{Functionality of the Generation and Application of the Data Spatialization Products Module}

It was specifically designed for multiple applications to observational data. Due to the dot characteristics of observational data, these data had relatively high precision and good representation. Nevertheless, data assimilation and coupling should be performed to monitor the process of crop growth on a large scale and include all the growth features of farmland crops. To realize data coupling, space-time information registration needs to be performed. Therefore, this module was established. It conducted data spatialization according to node and sensor type. The spatialization 
interpolation module that was designed provided, amongst other features, Inverse Distance to a Power, Kriging, and polynomial fitting.

\section{Key Technologies Involved in System Integration}

After comprehensive analysis and testing of this system, the system concentrated on farmland observational data flow processing and continuously handled received data by converting original binary files into visual and usable advanced outputs. Several key technologies should be comprehensively employed including database technology, data correction technology and data assimilation and interpolation technology.

\subsection{Database Technology}

This database involved a relatively large data set originating from a wide range of sources and had discord between time and space resolution. To better organize and utilize the data collected, this system utilized Oracle $10 \mathrm{~g}$ as a database platform and employed metadata technology to establish a database structure, proper handling of a substantial amount of abnormal data and effectively resolving data organization and storage-related problems.

In the current system, each node acquired a frame of observational data every 10 min(including photo and video clips). At the same time, basic geographical information, statistical data, and partial remote sensing data were also collected. Therefore, data abnormalities and the expansion principle should be taken into account when designing a system module. A plausible module should be created to minimize data storage redundancy, enhance data compatibility, realize data sharing, guarantee data security and browse and handle data efficiently.

The database performed data browsing using standard metadata and put in a highcapacity binary file that was written into the metadata field to enhance browsing speed. Additionally, it could undertake data addition, revision, deletion and browsing based upon metadata technology which accelerated data browsing and digging. During the process of metadata field programming and designing, international standard principles were followed and the procedure aimed to comply with current industrial, state, and international standards. This design procedure also considered the specific requirements of the data characteristics and utilization in this database[14]. A total of 12 fields were designed for metadata. Under the prerequisite of satisfying data enquiry and complete data description, a minimum number of data fields were designed to ease the complexity of metadata fill-in and avert data redundancy.

All storage data were upgraded to perform enquiry, statistical and comprehensive analysis. For real-time acquired farmland indicators and data, correction data were also retained as well as original current values. Both original graphics and inversion outputs were saved for graphics data. Regarding graded outputs from obtained data, relevant regulations were enforced about file names and the evolutionary process of 
pertinent data were described by using data dictionary technology[15]. In addition, dictionary information was used to establish data indices and increase the efficiency of data utilization.

\subsection{Data Correction Technology}

In this system, data correction had two correction stages. The first step was data conversion, which corrected the WSNs' deprived, raw and binary data by converting a photoelectricity signal into standard data showing temperature, humidity, and photosynthesis. The second step was to delete abnormal values and mark them as special data for the purpose of averting the negative influence that using these abnormal data can have on overall data quality.

Data correction flow: 1). Data value conversion: The front-end data sensor used in this study was purchased from the open market. These sensors were corrected under a constant temperature/humidity environment before delivery and the correction coefficient was kept in a standard register. The correction coefficient automatically corrected the signals emitted from sensors and simultaneously exported the measured value of digital quantity via a data bus. These initial measured values were mere 'relative values', which required linear and temperature offset correction. These relative data were in binary data format when sent back from the wireless sensor (bit stream). Prior to data browsing and storage, these data should be converted into another format[9]. Microsoft office Excel 2003 format file was commonly employed. The daily data obtained were saved, according to file acquisition date, as one single file with an extension name of .xls. The data correction procedure was located in the data automatic storage system. A preliminary version of the observational data was created following data storage into the database. 2). Abnormal data handling: Certain large errors or significantly abnormal data may occur that can be caused by impaired sensors and weak photoelectric signals. For example, among the data measured in Hebi city test region, some data showed that soil temperature was below minus $20^{\circ} \mathrm{C}$ or above $30^{\circ} \mathrm{C}$, which were obviously wrong. Those sorts of abnormal temperature/humidity values should be deleted even after data conversion. Otherwise, obvious calculation errors may occur in subsequent analysis (statistical analysis and spatial interpolation value analysis), leading to misleading and inaccurate monitoring outcomes. Therefore, the second step was indispensable. The procedure was undertaken in the database management and maintenance module. The corrected data were the revised version of the original observational data, which could be regarded as reliable raw observational values.

\subsection{Data Assimilation and Interpolation Technology}

To make coordinate use of background data from other test zones and real-time farmland field indicators, obtained data were subjected to further processing enabling different data to have comparable space-time nodes. The first step was to spatialize obtained data using data assimilation and interpolation technology. The modules used during interpolation included weighing interpolation, Kriging, and polynomial fitting methods. 
This project innovatively improved the interpolation procedure the system used in this project, performing orientation interpolation assisted by remote sensing graphics and basic geographical data about the test zone. The basic principle and steps were described as follows. Firstly, the plantation graph was extracted depending upon the land-use map and remote sensing graphics of the current period. Through observing GPS information at each observation point, interpolation modules were selected for formal interpolation in the region where there was uniform planting. The interpolation technology is detailed in Fig.4.

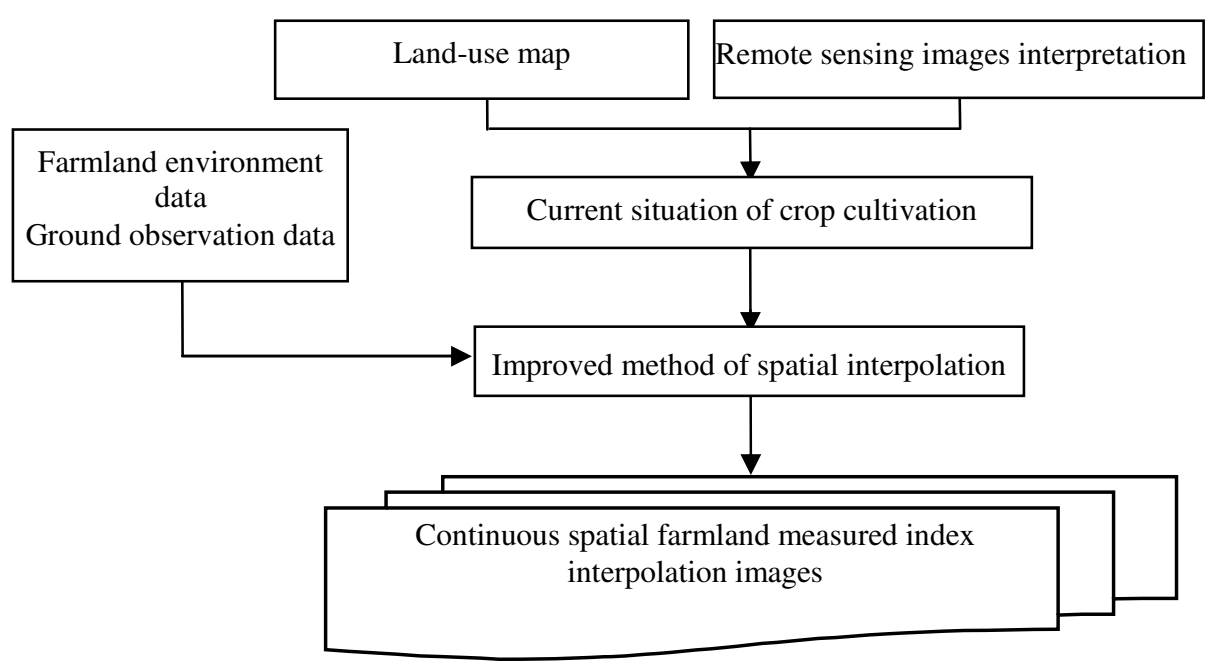

Fig. 4. Farmland observational data interpolation flow

\section{Realization and Application of the System}

After reviewing the system's targets and requirements, a detailed design was undertaken. All the potential data in the system were cleaned and analyzed and a corresponding database module was established. An Oracle 10g software platform, designed for business, was employed to set up the database. Microsoft Visual Studio 2005 language was used for system modeling and arithmetic packaging and a humanmachine interface was designed. Partial function dynamic databases of ESRI ArcGIS Engine 9.3, amongst others, were used in module integration.

During the process of system integration, an object-oriented method was adopted. The integration system first separated the database from the software system, ensuring universality among various software packages. At the same time, under an objectoriented human-machine operation environment, an instrument panel-like data input interface was designed, providing convenience to customers and allowing visual data browsing. A visually friendly interface was designed in the human-machine dialog box. To enhance utilization efficiency of the system, complex and simple operations were automated, allowing unmanned automatic operation. The system products that 
have been designed have attractive interfaces and are easy to operate and maintain, e.g. the data input interface was designed as the dialog box shown in Figure.5.

All the data obtained in the Hebi test zone, Henan province, were properly managed using this system. Approximately 5040 real-time monitoring data points were acquired daily and then stored in the database automatically. Since the system was deployed in July 2010, it has operated stably and reliably. WSN-acquired data and farmland information from other test zones were utilized to produce multiple forms of data output, that help monitor agricultural conditions. The system particularly played a vital role during the monitoring process of drought damage caused to winter wheat in December, 2010.

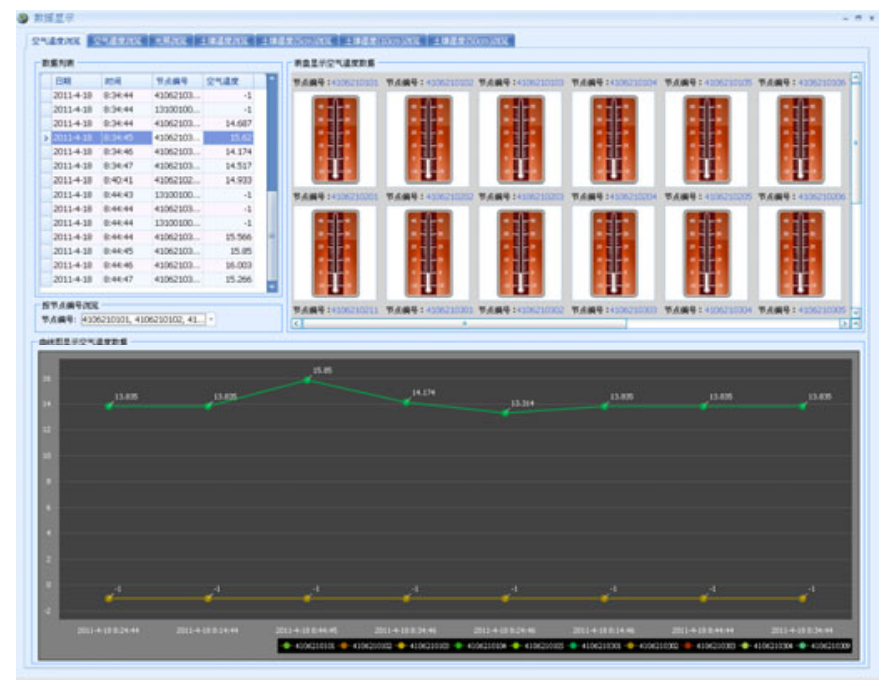

Fig. 5. Database storage module

\section{Conclusion}

This study focuses on the processing procedure of WSNs-acquired farmland data. It systematically analyses the information flow during the reception, storage, and analysis processes from farmland data collection. Additionally, this study designed a relevant database module that organized and managed a large amount of collected data. Pertinent software exploration platforms were employed to develop a desirable system for farmland observational data processes.

Application results reveal that software data flow and the integrated module design concept are good. The system has excellent stability and an attractive and simple human-machine interface. It also preliminarily achieves the task of monitoring data flow throughout the whole procedure from acquisition to utilization of the farmland information. Metadata and data dictionary dual techniques were employed in data 
organization, which improves data browsing and analysis efficiency. At the same time, a commonly used spatial interpolation module had improvements made to its monitoring data spatialization so that there was an increase in the precision of spatial interpolation values. To conclude, the system designed in this study preliminarily achieved the management and utilization functions of WSN-acquired farmland information, supplying an information service for agricultural field monitoring.

Acknowledgments. Funding project: National development and planning project for high-tech research of China entitled Remote sensing farmland information based on satellite and land in coordination with inversion technology (grant No. 2009AA12Z143). Surface project of National Natural Science Foundation of China entitled Simulation research on crop yield per unit based on space-time coupling and data assimilation (grant No. 40971218). And foundation of central institutes(iarrp2011- 15).

\section{References}

1. Cao, W., Yang, B., Pei, Z., et al.: Investigation and analysis of agricultural condition information demand in China. Transactions of the CSAE 20(1), 147-151 (2004)

2. Li, J., Zhang, J., Guan, H.: The technology development of agricultural condition information Both Abroad and Home. Science and Technology of Tianjin Agriculture and Forestry 4(2), 36-39 (2000)

3. Mu, L., Liu, G., Huang, J.: Design of farm field data collection and transmission system based on Java phone. Transactions of the CSAE 22(11), 165-169 (2006)

4. Gong, P.: Wireless sensor network as a new ground remote sensing technology for environmental monitoring. Journal of Remote Sensing 11(4), 545-551 (2007)

5. Song, L., Li, J., Chen, Y., et al.: Wireless Sensor Networks. Tsinghua University Press, Beijing (2005)

6. Mao, X., Yang, M., Mao, D.: Survey on wireless sensor network applications. Computer Applications and Software 25(3), 179-181 (2008)

7. Yang, Y., Zhao, J., Yi, W.: Iterative Joint Source Channel Decoding For Multimedia Wireless Sensor Networks. CWSN, 12-15 (2008)

8. Liu, C., Zhao, J., Yi, W.: Design of wireless image sensor node based on nRF24L01. Electronic Measurement Technology 31(6), 136-139 (2008)

9. Gao, F., Yu, L., Wang, Y., et al.: Development of host computer software for crop water status monitoring system based on wireless sensor networks. Transactions of the CSAE 26(5), 175-181 (2010)

10. Feng, G., Li, Y., Zhang, W., et al.: Research and design of crop water status monitoring system based on wireless sensor networks. Transactions of the CSAE 25(2), 107-112 (2009)

11. Cai, Y., Liu, G., Li, L., et al.: Design and test of nodes for farmland data acquisition based on wireless sensor network. Transactions of the CSAE 25(4), 176-178 (2009)

12. Li, X., Sun, Z., Xiao, C., et al.: Development of remote monitoring system based on $\mu \mathrm{C} / \mathrm{OS}-\mathrm{II}$ embedded technology for agricultural environment. Transactions of the CSAE 23(10), 156-161 (2007) 
13. Li, X., Sun, Z., Huang, T.: Study on application of embedded system for the agricultural information acquisition based on WEB. Agriculture Network Information (12), 33-37 (2005)

14. Xiong, J., Li, Z., Chen, P., et al.: Design and implementation of metadata-based technology platform for data sharing. Microcomputer \& It's Applications (9), 13-16 (2010)

15. Fu, Y., Tian, Z., Zhao, X., et al.: Two applications of oracle DD basing on VBA and ASP. Computer Engineering and Applications (6), 178-181 (2005) 